\title{
COMPARAÇÃO ENTRE MÉTODOS COMPOSTOS NO CÁLCULO DE AFINIDADES POR PRÓTON E ELÉTRON EM SISTEMAS MOLECULARES
}

\author{
José Carlos B. de Lima e Nelson H. Morgon* \\ Instituto de Química, Universidade Estadual de Campinas, CP 6154, 13083-970 Campinas - SP, Brasil
}

Recebido em 29/10/08; aceito em 19/6/09; publicado na web em 27/11/09

\begin{abstract}
COMPARISON AMONG COMPOSITE METHODS ON THE CALCULATION OF PROTON AND ELECTRON AFFINITIES IN MOLECULAR SYSTEMS. The CBS-4M, CBS-QB3, G2, G2(MP2), G3 and G3(MP2) model chemistry methods have been used to calculate proton and electron affinities for a set of molecular and atomic systems. Agreement with the experimental value for these electronic properties is quite good considering the uncertainty in the experimental data. A comparison among the six theories using statistical analysis (average value, standard deviation and root-mean-square) showed a better performance of CBS-QB3 to obtain these properties.
\end{abstract}

Keywords: composite methods; CBS-QB3 method; proton and electron affinities.

\section{INTRODUÇÃO}

A partir da década de 1980 começaram a surgir métodos para obter a energia eletrônica de sistemas moleculares, utilizando cálculos teóricos em diferentes níveis de teoria e com conjuntos de funções de base específicos para cada um desses níveis. Estes métodos ficaram conhecidos como métodos compostos. A principal idéia por trás destes métodos é obter a energia eletrônica na geometria de equilíbrio de forma aditiva, ou seja, calculada pela soma das contribuições de cada etapa do cálculo.

Existem diversos métodos computacionais, sendo os mais utilizados aqueles que derivam da teoria Gaussian desenvolvidos por Pople e colaboradores ${ }^{1-4}$ e da teoria do Conjunto de Base Completo desenvolvidos por Petersson e colaboradores. ${ }^{5,6}$

Usando a energia do sistema é possível obter propriedades termodinâmicas como a entalpia e a energia livre de Gibbs. Assim, através da variação da entalpia de uma reação é possível o cálculo de propriedades como afinidade por próton e por elétron. Além disso, podem ser obtidas propriedades eletrônicas e estruturais de cálculos teóricos como densidade de carga, comprimento e ângulo de ligação.

Os métodos compostos podem ser utilizados, por exemplo, na determinação de mecanismos de reações utilizando as propriedades termodinâmicas no cálculo da energia de ativação e de constantes de velocidade, ${ }^{7-14}$ úteis em pesquisas que examinam em detalhes o mecanismo através do cálculo de constantes de velocidade para cada etapa da reação. ${ }^{15,16}$

Outras propriedades muito estudadas são as afinidades por próton ${ }^{17-23}$ e por elétron, ${ }^{24-27}$ a acidez ${ }^{28}$ bem como a identificação da estrutura mais estável para um determinado isômero. ${ }^{29,30}$ Compostos inorgânicos também podem ser explorados utilizando métodos compostos pelo cálculo do potencial de redução ${ }^{31}$ em reações de óxido-redução.

No trabalho de um químico, informações a respeito de entalpia de formação e energia de dissociação são muito importantes, existindo diversos estudos neste campo, onde os métodos compostos são amplamente utilizados. ${ }^{32-44}$ Outra aplicação interessante é no estudo do mecanismo em processos envolvidos em espectroscopia de massas. ${ }^{45}$

A variedade de aplicações utilizando métodos compostos vem aumentando tanto em trabalhos teóricos como em trabalhos expe-

*e-mail: morgon@iqm.unicamp.br rimentais, devido à sua praticidade, confiabilidade e precisão em prever parâmetros físico-químicos. Este trabalho avaliou alguns métodos compostos considerando-se a capacidade de reprodução de parâmetros experimentais e o custo computacional para a obtenção dos mesmos. O processo de validação foi realizado através da análise estatística de um conjunto de sistemas atômicos e moleculares.

\section{Métodos compostos}

Os métodos compostos, de forma geral, sofreram várias modificações com o decorrer do tempo para a solução de problemas anteriores e descrição de novos sistemas. ${ }^{46,47}$ Isto pode ser observado na sequência de trabalhos dos quais surgiram, por exemplo, os métodos CBS-4M ${ }^{6,48}$ e CBS-QB3, ${ }^{5}$ bem como os métodos G2, ${ }^{1} \mathrm{G} 2(\mathrm{MP} 2),{ }^{2} \mathrm{G} 3{ }^{3}$ e G3(MP2). ${ }^{4}$ Este trabalho apresentará em detalhes como o método CBS-QB3 obtém a energia molecular total pelo fato de ter apresentado uma maior eficiência na obtenção das propriedades estudadas.

\section{O método $C B S-Q B 3$}

A descrição quântica de uma propriedade química será mais precisa quanto mais o cálculo da correlação eletrônica se aproximar do nível full-CI e quanto mais completo for o conjunto de funções de base utilizado na representação eletrônica do sistema. ${ }^{46} \mathrm{Um}$ cálculo full-CI seria obtido utilizando um conjunto completo de determinantes de Slater gerados pela distribuição de todos os elétrons em todos os orbitais do sistema. ${ }^{49}$ Entretanto, quanto mais completa a representação do sistema mais complexa é sua resolução.

A descrição completa da função de onda e a utilização de conjunto de funções de base cada vez maiores fazem com que a convergência seja mais lenta, portanto maior será o custo computacional. A solução deste problema pode ser obtida através da extrapolação da base, ${ }^{32,46,47}$ caminho utilizado nos métodos de conjunto de base completo (CBS). Outra possibilidade é o uso de um conjunto de funções de base o maior possível, bem como da propriedade aditiva na obtenção da energia molecular, solução utilizada nos métodos Gaussian- $n .^{50}$

A idéia principal dos métodos de conjunto de base completo é a otimização da geometria e obtenção da energia do ponto zero (ZPE) através de cálculos HF ou B3LYP. O próximo passo é uma série de cálculos single-point de alto nível da energia eletrônica na geometria de equilíbrio. Utiliza-se um grande conjunto de funções de base para os níveis mais simples de teoria, conjunto de funções de base médio 
para cálculos MP2, e conjunto de funções de base pequeno para teorias de alto nível. O nível superior de uma teoria está relacionado com a maior precisão no cômputo da correlação eletrônica que é obtida através de cálculos single-point..$^{47}$

O método CBS-QB3 inicia-se com a otimização no nível B3LYP utilizando o conjunto de funções de base $6-311 \mathrm{G}(2 \mathrm{~d}, \mathrm{~d}, \mathrm{p}),{ }^{5,51}$ neste nível são calculadas também as frequências vibracionais, a energia do ponto zero, bem como a entropia do sistema e a correção térmica. Posteriormente são realizados cálculos single-point no nível coupledcluster - CCSD(T) - com o conjunto de funções de base 6-31+G(d'), no nível Møller-Plesset de quarta ordem com excitações simples, duplas e quádruplas - MP4(SDQ) - com o conjunto de funções de base $6-31+\mathrm{G}((\mathrm{d}) \mathrm{f}, \mathrm{p})^{51}$ e, por fim, no nível Møller-Plesset de segunda ordem - MP2 - com o conjunto de funções de base 6-311+G(2df), realizando neste nível a extrapolação da base para que o resultado seja obtido num conjunto de funções de base completo. ${ }^{5,48}$

A energia eletrônica CBS-QB3 a zero kelvin - CBS-QB3 (0K) - é obtida através da Equação 1, que já inclui a energia do ponto zero escalonada, representada por $\Delta \boldsymbol{E}(\boldsymbol{Z P E})$.

$C B S-Q B 3(O K)=E(M P 2)+\Delta E(Z P E)+\Delta E(C B S)+\Delta E(E M P)+$ $\Delta E(S P I N)+\Delta E(M P 4)+\Delta E(C C S D)+\Delta E($ Int $)$

O termo $\boldsymbol{E}(\boldsymbol{M P 2})$ é a energia Møller-Plesset de segunda ordem. O cálculo é realizado desconsiderando os elétrons internos, pois os efeitos de correlação eletrônica gerados pelas interações caroçocaroço e caroço-valência não interferem de maneira significativa no valor da variação de energia obtida para os átomos do primeiro período, com exceção do sódio. .6,48 $^{4}$

O termo $\Delta \boldsymbol{E}(\boldsymbol{Z P E})$ corresponde à correção da energia do ponto zero escalonada, é obtido pela multiplicação da correção do ponto zero (Zero-point correction) do cálculo B3LYP pelo fator de escalonamento $(\lambda)$ 0,99 (Equação 2).

$\Delta E(Z P E)=Z P E . \lambda$

O termo $\boldsymbol{\Delta} \boldsymbol{E}(\boldsymbol{C B S})$ inclui a correção da energia MP2 devido a extrapolação da base (Equação 3).

$\Delta E(C B S)=E^{(2)}-E(C B S)$

onde o parâmetro $E^{(2)}$ corresponde à energia de correlação MP2 para um par de elétrons (Equação 4).48

$E^{(2)}=\sum_{i, j}^{o c c} e_{i j}^{(2)}$

com

$e_{i j}^{(2)}=\sum_{a, b}^{v i r t} C_{i j}^{a b} V_{i j}^{a b}$

representando a energia da correlação, e pode ser obtida pelo produto dos elementos da matrix hamiltoniana acoplando os orbitais ocupados $\psi_{\mathrm{i}}$ e $\psi_{\mathrm{j}}$ aos orbitais virtuais $\psi_{a}$ e $\psi_{b}$. $V_{i j}{ }^{a b}$ e $C_{i j}{ }^{a b}$ são descritos pelas seguintes equações:

$V_{i j}^{a b}=\left\langle\psi_{i} \psi_{j}\left|\frac{1}{r_{12}}\right| \psi_{a} \psi_{b}\right\rangle$

$C_{i j}^{a b}=\frac{V_{i j}^{a b}}{\left[e_{i}+e_{j}-e_{a}-e_{b}\right]}$

Ainda na Equação 3, o parâmetro $E(C B S)$ representa a energia de correlação MP2 para um par de elétrons obtida através do formalismo matemático que representa a extrapolação da base (Equação 6).
$E(C B S)=E 2\left(l_{\max 2}\right)+\left[E 2\left(l_{\max 2}\right)-E 2\left(l_{\max 1}\right)\right] \times\left[\frac{\left(l_{\max 2}+\frac{1}{2}\right)^{-3}}{\left(l_{\max 1}+\frac{1}{2}\right)^{-3}-\left(l_{\max 2}+\frac{1}{2}\right)^{-3}}\right](6)$

com E2 $\left(1_{\max 2}\right)$ representando a energia MP2 obtida com o conjunto de funções de base cc-pVTZ e E2 $\left(1_{\text {maxx }}\right)$ a energia MP2 obtida com o conjunto de funções de base cc-pVDZ ${ }^{47}$ A extrapolação é realizada de forma a reduzir a valor quadrático médio entre o valor experimental e calculado para a entalpia de 17 reações. ${ }^{32}$

Duas correções empíricas são adicionadas no método CBSQB3, ${ }^{5,46,48}$ um parâmetro de um elétron e outro de dois elétrons. O parâmetro de dois elétrons - $\Delta \boldsymbol{E}(\boldsymbol{E M P})$ - corrige o erro gerado pela diferença entre a energia MP2 calculada e os valores experimentais (Equação 7).

$\Delta E(E M P)=-5,79 \sum_{i=1}^{n_{\beta}}\left[\sum_{\mu=1}^{N_{v i r}+1} C_{\mu_{i i}}\right]^{2}|S|_{i i}^{2}$

Sendo:

$|S|_{i i}=\int\left|\varphi_{i}^{\alpha} \varphi_{i}^{\beta}\right| d \tau$

a integral de recobrimento absoluta entre os $\alpha$ - e $\beta$-spin orbitais mais similares e:

$\left[\sum_{\mu=1}^{N_{\text {vir }}+1} C_{\mu_{i i}}\right]^{2}$

o fator de interferência, que é obtido pelo quadrado do traço da função de onda Møller-Plesset de primeira ordem. O termo $C \mu_{\mathrm{ii}}$ da Equação 9 é o mesmo descrito para a Equação $5\left(C_{\mathrm{ij}}\right.$ b) , porém neste caso tratase somente da diagonal da matriz de coeficientes da função de onda de primeira ordem. Para uma descrição mais detalhada consultar as refs. 52 e 53. Este fator está relacionado com a energia de correlação resultante da interação de Coulomb de pares eletrônicos. ${ }^{52-55} \mathrm{O}$ coeficiente $-5,79$ (em mili-hartree) ${ }^{56}$ é obtido minimizando a valor quadrático médio para 55 energias de dissociação experimentais. ${ }^{5}$

O parâmetro de um elétron - $\Delta \boldsymbol{E}(\boldsymbol{S P I N})$, aplicado somente a sistemas de camada aberta, incorpora uma correção eletrônica para a contaminação spin gerada pelo fato da função de onda UHF de primeira ordem, utilizada para esses cálculos, não ser autofunção do operador $\left\langle\mathrm{S}^{2}\right\rangle,{ }^{57-59}$ dessa forma a correção é feita pelo erro gerado em $\left\langle\mathrm{S}^{2}\right\rangle\left(\Delta\left\langle\mathrm{S}^{2}\right\rangle\right)$ (Equação 10).

$\Delta E(S P I N)=-9,54 \Delta\left\langle S^{2}\right\rangle$

O coeficiente -9,54 (em mili-hartree) ${ }^{56}$ é obtido pela minimização da valor quadrático médio para os valores experimentais de energia de dissociação, potencial de ionização e da afinidade por elétron para alguns sistemas químicos. ${ }^{5}$

Outra correção devido à interferência da extrapolação da base é incluída através do fator $\boldsymbol{\Delta E}$ (Int) (Equação 11).

$\Delta E($ Int $)=\Delta E^{(\infty)}-\Delta E^{(2)}$

Na Equação 11, $\Delta E^{(2)}$ representa a correção do conjunto de base completo (CBS) total para a energia de correlação de segunda ordem utilizando MP2, e $\Delta E^{(\infty)}$ a correção CBS total para a correlação eletrônica full-CI aproximada - utilizando CCSD(T). A diferença entre as correções $\operatorname{CCSD}(\mathrm{T})$ e de segunda ordem incorpora o truncamento do conjunto de funções de base e uma correção da interferência CBS para componentes de alto nível da correlação eletrônica no cálculo da energia. ${ }^{48,52,53}$ 
O termo $\boldsymbol{\Delta} \boldsymbol{E}(\boldsymbol{M P 4})$ (Equação 12) inclui efeitos de correlação superiores à energia Møller-Plesset de segunda ordem, bem como adiciona o efeito da utilização de funções de polarização no conjunto de funções de base.

$$
\Delta E(M P 4)=E[M P 4(S D Q) / 6-31+G(d(f), p)]-E[M P 2 / 6-31+
$$$$
g(d(f), p]
$$

Por último, o termo $\Delta \boldsymbol{E}(\boldsymbol{C C S D})$ (Equação 13) inclui efeitos de correlação superiores à energia Møller-Plesset de quarta ordem MP4(SDQ) - corrigindo também resíduos de contaminação spin.

$\Delta E(C C S D)=E\left[C C S D(T) / 6-31+\left(d^{\prime}\right)\right]-E[M P 4(S D Q) / 6-31+$ $\left.\left(d^{\prime}\right)\right]$

Assim, através da otimização da geometria molecular em $\boldsymbol{B} 3 \mathbf{L Y P}$ são obtidos os termos energia do ponto zero, entropia e a correção térmica. Com o cálculo single-point MP2 obtém-se os termos $\mathbf{E}(\mathbf{M P 2}), \Delta \mathbf{E}(\mathbf{C B S}), \Delta \mathbf{E}(\mathbf{E M P}), \Delta \mathbf{E}(\mathbf{S P I N})$ e $\Delta \mathbf{E}$ (Int); com o cálculo single-point MP4(SDQ) o termo $\triangle \mathbf{E}(\mathbf{M P 4})$ e com o cálculo singlepoint $\mathbf{C C S D}(\mathbf{T})$ o termo $\Delta \mathbf{E}(\mathbf{C C S D})$, necessários para o cálculo da entalpia e da energia livre CBS-QB3.

Com a entalpia e a energia livre é possível explorar uma variedade de propriedades termodinâmicas como acidez, basicidade, energia de ionização, afinidades por próton e por elétron, entre outras. ${ }^{45}$ Este trabalho faz uso da afinidade por próton e por elétron para o processo de validação, as quais serão abordadas na próxima seção.

\section{PROPRIEDADES TERMODINÂMICAS}

\section{Afinidade por elétron}

\section{Afinidade por elétron atômica}

A afinidade por elétron (AE) é definida como a energia necessária para remover um elétron de um anion $\left(M^{-}\right)$, e pode ser obtida pela variação da energia total $(\Delta E)$ (Equação 14$){ }^{60-62}$

$\mathrm{M}^{-} \rightarrow \mathrm{M}+\mathrm{e}^{-} \mathrm{AE}=\Delta \mathrm{E}(0 \mathrm{~K})$

Portanto, a diferença entre a energia total de um átomo $M\left(E_{M}\right)$ e a energia total do seu íon $\left(E_{M^{-}}\right)$, a zero kelvin, é considerada como sendo a afinidade por elétron do átomo $M$ (Equação 15).

$\mathrm{AE}_{(\mathrm{M})}=\mathrm{E}_{\mathrm{M}}-\mathrm{E}_{\mathrm{M}}-(0 \mathrm{~K})$

O valor da afinidade por elétron pode fornecer informação sobre a estabilidade de ânions. Valores positivos de afinidade indicam, por exemplo, que o ânion é estável. ${ }^{63}$

\section{Afinidade por elétron molecular}

A afinidade por elétron de uma molécula neutra é considerada como a energia de ligação de um elétron na molécula. Isto pode ser comparado teoricamente com a obtenção experimental de $\mathbf{A E}$ através de uma transição espectroscópica ${ }^{60-62}$ (Equação 16).

$\mathrm{AE}_{(\mathrm{M})}=\Delta \mathrm{E}\left(\mathrm{M} \leftarrow \mathrm{M}^{-}\right)$

A afinidade por elétron molecular é, portanto, considerada como a energia de transição do estado fundamental do ânion para o estado fundamental da espécie neutra. É possível obtê-la teoricamente através do cálculo da energia total da molécula neutra na geometria otimizada $\left(E_{M}\right)$ e da energia total do ânion também na geometria otimizada $\left(E_{M}{ }^{-}\right)$ a zero kelvin (Equação 17).
$\mathrm{AE}=\mathrm{E}_{\mathrm{M}}-\mathrm{E}_{\mathrm{M}^{-}}(\mathrm{OK})$

A afinidade por elétron é uma importante propriedade termodinâmica, sendo extensamente estudada para diversas aplicações onde a transferência de elétron promove determinada reação. ${ }^{64-66}$ As aplicações vão desde da área biomédica ${ }^{17,67,68}$ ao estudo de propriedades eletrônicas de compostos inorgânicos com importantes implicações teóricas ou práticas, ${ }^{69-73}$ inclusive no estudo de nanopartículas. ${ }^{74-76}$

\section{Afinidade por próton}

A afinidade por próton (AP) pode ser definida de forma geral como a reação, em fase gasosa, de um próton $\mathrm{H}^{+}$e um sistema químico qualquer $(M)$, normalmente um sistema neutro, ${ }^{77} \mathrm{e}$ ser obtida através do negativo da variação da entalpia $(\Delta H){ }^{45,63,78-81}$ Considerando, por exemplo, a afinidade por próton de um sistema aniônico $\left(M^{-}\right)$, a reação será representada pela Equação 18 a 298 kelvin. ${ }^{17}$

$\mathrm{M}^{-}+\mathrm{H}^{+} \rightarrow \mathrm{HM} \quad \mathrm{AP}=-\Delta_{\mathrm{r}} \mathrm{H}(298 \mathrm{~K})$

Outra convenção representa a $\mathbf{A P}$ como a retirada de um próton de um sistema qualquer: $\mathbf{H M}{ }^{\circledR} \mathbf{M}^{-}+\mathbf{H}^{+}$, neste caso a $\mathbf{A P}=+\Delta \mathrm{H}_{\mathrm{r}}{ }^{63}$ Portanto, a diferença entre a entalpia do sistema neutro $\left(H_{H M}\right)$ e do sistema aniônico $\left(H_{M^{-}}\right)$é considerada como sendo a afinidade por próton do íon $M^{*}$ (Equação 19).

$\mathrm{AP}_{\mathrm{M}^{-}}=-\left(\mathrm{H}_{\mathrm{HM}}-\mathrm{H}_{\mathrm{M}^{-}}\right)$

O estudo da afinidade por próton é aplicado a processos químicos onde ocorrem transferências de próton. Trata-se de um parâmetro importante em estudos biomédicos. ${ }^{82-84}$ Também é utilizado para estudar compostos inorgânicos ${ }^{85-87}$ bem como de outros tipos de espécies químicas ${ }^{88-90}$.

\section{METODOLOGIA COMPUTACIONAL}

A análise estatística para averiguar a capacidade dos métodos compostos em reproduzir valores experimentais foi realizada pelos cálculos do erro absoluto, do valor médio, do desvio padrão e da raiz média quadrática. ${ }^{91}$ Avaliou-se também o custo computacional observando-se o método composto que concluía o cálculo no menor tempo.

A escolha de métodos compostos deve-se ao baixo custo computacional e à confiabilidade nos resultados. Para a afinidade por próton espera-se uma precisão de $8,4 \mathrm{~kJ} / \mathrm{mol}$ (ou $2 \mathrm{kcal} / \mathrm{mol}$ ) e para a afinidade por elétron uma precisão de $0,1 \mathrm{eV}$ entre o valor calculado e experimental. ${ }^{92}$ A obtenção da energia molecular foi realizada utilizando o programa Gaussian nas versões (98) e (03)..$^{93,94}$

Para a afinidade por próton estudaram-se 16 sistemas: $\mathrm{CH}_{3} \mathrm{CH}_{2} \mathrm{NH}^{-}$, $\mathrm{CH}_{3} \mathrm{CH}_{2} \mathrm{O}^{-}, \mathrm{CH}_{3} \mathrm{COO}^{-}, \mathrm{CH}_{2} \mathrm{CCNO}^{-}, \mathrm{HSO}_{4}^{-}, \mathrm{HOO}-\mathrm{CH}_{2}^{-}, \mathrm{BH}_{2}^{-}, \mathrm{NH}^{-}$, $\mathrm{NH}_{2}^{-}, \mathrm{NO}_{2}^{-}, \mathrm{HO}^{-}, \mathrm{O}_{2}^{-}, \mathrm{O}^{-}, \mathrm{S}^{-}$e $\mathrm{S}_{2}^{-}$. Para a afinidade por elétron estudaram-se 24 sistemas (a multiplicidade é indicada entre parênteses): $\mathrm{C}, \mathrm{NH}, \mathrm{HNO}$ e $\mathrm{SO}_{2}$ (estado singleto); $\mathrm{ClCH}_{2} \mathrm{COO}, \mathrm{CH}_{3} \mathrm{CH}_{2} \mathrm{NH}, \mathrm{CH}_{3} \mathrm{CH}_{2} \mathrm{O}, \mathrm{CH}_{3} \mathrm{COO}$, $\mathrm{CH}_{2} \mathrm{COCN}, \mathrm{C}_{2} \mathrm{H}, \mathrm{H}_{2} \mathrm{~S}, \mathrm{HOO}, \mathrm{B}_{2} \mathrm{~N}, \mathrm{BH}_{2}, \mathrm{CH}, \mathrm{CH}_{2}, \mathrm{NH}_{2}, \mathrm{OH}, \mathrm{NO}_{2}, \mathrm{SH}$ e $\mathrm{PH}_{2}$ (estado dupleto); e $\mathrm{O}_{2}, \mathrm{~S}_{2}$ e $\mathrm{PH}$ (estado tripleto). A escolha dos estados eletrônicos está relacionada à multiplicidade dos sistemas utilizados para a obtenção experimental da $\mathrm{AE}\left(\mathrm{O}_{2},{ }^{95} \mathrm{~B}_{2} \mathrm{~N},{ }^{96} \mathrm{SO}_{2},{ }^{97}\right.$ outros $\left.{ }^{98}\right)$.

\section{RESULTADOS E DISCUSSÃO}

\section{Validação do método}

Uma forma de examinar o método computacional mais adequado para representar uma reação, a fim de estudar o mecanismo 
pelo qual ela se processa, é comparando os valores calculados com os valores experimentais para determinadas propriedades termodinâmicas. Uma análise estatística permite validar o método que melhor representa os valores experimentais. A metodologia mais adequada para o estudo da reação foi escolhida através de uma validação entre os métodos CBS-4M, CBS-QB3, G2, G2(MP2), G3 e G3(MP2) disponíveis no programa Gaussian. As propriedades estudadas neste trabalho foram a afinidade por próton e por elétron, observando-se também o método que gerou os resultados no menor tempo de CPU.

Nas Tabelas 1 e 2 encontram-se os valores experimentais ${ }^{98}$ e calculados para as afinidades por próton $(\mathrm{em} \mathrm{kJ} / \mathrm{mol})$ e por elétron $(\mathrm{em} \mathrm{eV})$, respectivamente, bem como os valores para o valor médio $\left(\mathrm{V}_{\mathrm{m}}\right)$, desvio padrão $\left(\delta_{\mathrm{N}-1}\right)$ e valor quadrático médio (RMS).

A Tabela 1 apresentou a seguinte ordem para os valores de RMS: G3 〈CBS-QB3 > G2(MP2) < G2 < G3(MP2) < CBS-4M. Portanto, para a AP observou-se que o menor valor de RMS foi obtido para o método G3, porém este superestimou na média o valor experimental. O próximo melhor resultado foi para o método CBS-QB3, porém apresentando um valor coerente para o valor médio. A pior performance foi observada para o método CBS-4M, o qual apresentou um alto valor para RMS.

A Tabela 2 apresentou a seguinte ordem para os valores de RMS: CBS-QB3 < G3 < G2 < G3(MP2) < G2(MP2) < CBS-4M. Portanto, para a $\mathbf{A E}$ observou-se que o menor valor de RMS foi obtido para o método CBS-QB3, apresentando um valor coerente para o valor médio. Com exceção do método G3, os outros métodos apresentaram um alto valor para RMS.

\section{Eficiência dos métodos compostos no cálculo da energia}

A análise de algumas características inerentes a cada método permite explicar os resultados obtidos para a energia molecular. A correção empírica realizada nos métodos derivados da teoria Gaussian não é consistente em tamanho, uma consequência é que eles tendem a superestimar o resultado. ${ }^{46,99}$ A relação que representa esta correção é dada pela Equação 20.

$\Delta(H L C)=c_{2} n_{p a r}+c_{1}\left(n_{\alpha}+n_{\beta}\right)$

Nesta equação $(H L C)$ é chamado de "correção de alto nível", $n_{\alpha}$ e $n_{\beta}$ são os números de elétrons de valência com spin $\alpha$ e $\beta$, respectivamente; $n_{p a r}$ é o número de pares eletrônicos intraorbital; $c_{1} \mathrm{e}$ $c_{2}$ são constantes de um e dois-elétrons escolhidos empiricamente.

No método CBS considera-se também o recobrimento dos spinorbitais, e não somente o número de orbitais duplamente ocupados, assim a correção empírica varia suavemente como, por exemplo, no cálculo da dissociação de uma ligação, ou seja, com o aumento da distância entre os átomos. ${ }^{99}$ Outra característica que afeta o cálculo da energia é o fato dos métodos Gaussian obterem as frequências, necessárias para calcular a correção do ponto zero, no nível HF/6$31 \mathrm{G}(\mathrm{d})$. Neste nível de teoria não são considerados efeitos de correlação, o cômputo desses efeitos poderia melhorar o resultado para a energia. ${ }^{1,3,92}$

Outra diferença fundamental entre os métodos CBS e Gaussian, que interfere no cálculo da energia, está no tratamento adotado para a consideração de efeitos de correlação dinâmica. A região ao redor

Tabela 1. Valores experimentais e calculados de afinidade por próton (em kJ/mol) para os 16 sistemas estudados, obtidos com os métodos CBS-4M, CBS-QB3, G2, G2(MP2), G3 e G3(MP2), bem como os resultados obtidos para o valor médio ( $\mathrm{V}_{\mathrm{m}}$ - obtido pela subtração entre os valores experimentais e calculados), o desvio padrão $\left(\delta_{\mathrm{N}-1}\right)$ e o valor quadrático médio (RMS)

\begin{tabular}{|c|c|c|c|c|c|c|c|}
\hline \multirow[b]{2}{*}{ SISTEMA } & \multicolumn{6}{|c|}{ Afinidade por Próton } & \multirow[b]{2}{*}{ G3(MP2) } \\
\hline & $\mathrm{EXP}^{\mathrm{a}} \pm \delta_{\mathrm{N}-1}$ & CBS-4M & CBS-QB3 & $\mathrm{G} 2$ & G2(MP2) & G3 & \\
\hline $\mathrm{H}_{3} \mathrm{CCH}_{2} \mathrm{NH}$ & $1671,0 \pm 4,6$ & 1669,4 & 1665,1 & 1666,7 & 1666,7 & 1669,0 & 1667,2 \\
\hline $\mathrm{H}_{3} \mathrm{CCH}_{2} \mathrm{O}$ & $1579,0 \pm 8,8$ & 1578,5 & 1578,8 & 1578,8 & 1580,0 & 1582,3 & 1583,2 \\
\hline $\mathrm{H}_{3} \mathrm{CCOO}$ & $1456,0 \pm 9,2$ & 1446,8 & 1447,1 & 1446,0 & 1447,6 & 1449,8 & 1451,6 \\
\hline $\mathrm{CH}_{2} \mathrm{CCNO}$ & $1441,0 \pm 8,8$ & 1432,1 & 1431,5 & 1434,1 & 1434,2 & 1434,2 & 1432,9 \\
\hline $\mathrm{HSO}_{4}$ & $1295,0 \pm 11$ & 1286,2 & 1289,7 & 1295,2 & 1298,0 & 1294,8 & 1297,4 \\
\hline $\mathrm{HOO}$ & $1574,9 \pm 2,5$ & 1571,0 & 1568,6 & 1564,6 & 1568,6 & 1569,0 & 1570,8 \\
\hline $\mathbf{C H}_{2}$ & $1710,4 \pm 1,3$ & 1716,4 & 1711,6 & 1710,1 & 1711,5 & 1715,1 & 1714,9 \\
\hline $\mathbf{B H}_{2}$ & 1723,0 & 1732,9 & 1725,7 & 1720,3 & 1721,4 & 1721,2 & 1720,4 \\
\hline $\mathrm{NH}$ & $1664,8 \pm 2,1$ & 1676,6 & 1670,4 & 1669,7 & 1673,0 & 1674,7 & 1676,7 \\
\hline $\mathbf{N H}_{2}$ & $1687,8 \pm 0,4$ & 1686,4 & 1688,1 & 1683,4 & 1682,3 & 1688,6 & 1685,4 \\
\hline $\mathrm{NO}_{2}$ & $1423,4 \pm 0,8$ & 1415,0 & 1414,2 & 1413,9 & 1418,5 & 1416,2 & 1420,7 \\
\hline $\mathrm{HO}$ & 1633,0 & 1629,2 & 1634,0 & 1624,7 & 1626,6 & 1631,3 & 1631,6 \\
\hline $\mathbf{O}_{2}$ & $1477,0 \pm 2,9$ & 1442,6 & 1468,7 & 1475,7 & 1482,8 & 1479,4 & 1482,8 \\
\hline $\mathrm{O}$ & 1601,0 & 1599,5 & 1603,2 & 1600,4 & 1606,3 & 1611,6 & 1616,0 \\
\hline S & $1466,1 \pm 1,3$ & 1475,9 & 1458,2 & 1463,6 & 1467,3 & 1463,3 & 1467,4 \\
\hline $\mathbf{S}_{2}$ & $1380,0 \pm 5,0$ & 1392,6 & 1381,1 & 1389,0 & 1391,1 & 1385,8 & 1387,5 \\
\hline $\mathrm{V}_{\mathrm{m}}$ & & 2,0 & 3,0 & 2,9 & 1,0 & $-0,2$ & $-1,4$ \\
\hline$\pm \delta_{\mathrm{N}-1}$ & & $\pm 11,5$ & $\pm 5,4$ & $\pm 5,4$ & $\pm 6,3$ & $\pm \mathbf{5 , 6}$ & $\pm 6,4$ \\
\hline RMS & & 11,3 & 5,8 & 6,0 & 5,9 & 5,4 & 6,3 \\
\hline
\end{tabular}

${ }^{a}$ Ref. 98. 
Tabela 2. Valores experimentais e calculados de afinidade por elétron (em eV) para os 24 sistemas estudados, obtidos com os métodos CBS4M, CBS-QB3, G2, G2(MP2), G3 e G3(MP2), bem como os resultados obtidos para o valor médio $\left(\mathrm{V}_{\mathrm{m}}\right)$, o desvio padrão $\left(\delta_{\mathrm{N}-1}\right)$ e o valor quadrático médio (RMS). A multiplicidade (MULT.) é indicada para cada sistema

\begin{tabular}{|c|c|c|c|c|c|c|c|c|}
\hline \multicolumn{9}{|c|}{ Afinidade por Elétron } \\
\hline SISTEMA & MULT. & EXP. $^{a} \delta_{N-1}$ & CBS-4M & CBS-QB3 & G2 & G2(MP2) & G3 & G3(MP2) \\
\hline $\mathrm{ClCH}_{2} \mathrm{COO}$ & 2 & $3,910 \pm \mathbf{0 , 1 6 0}$ & 4,251 & 3,865 & 4,111 & 4,111 & 4,074 & 4,066 \\
\hline $\mathrm{H}_{3} \mathrm{CCH}_{2} \mathrm{NH}$ & 2 & $0,620 \pm \mathbf{0 , 1 4 0}$ & 0,729 & 0,618 & 0,634 & 0,647 & 0,592 & 0,631 \\
\hline $\mathrm{H}_{3} \mathrm{CCH}_{2} \mathrm{O}$ & 2 & $1,712 \pm \mathbf{0 , 0 0 4}$ & 1,946 & 1,788 & 1,817 & 1,827 & 1,760 & 1,770 \\
\hline $\mathrm{H}_{3} \mathrm{CCOO}$ & 2 & $3,470 \pm \mathbf{0 , 0 1 0}$ & 3,625 & 3,268 & 3,482 & 3,484 & 3,433 & 3,427 \\
\hline $\mathrm{CH}_{2} \mathrm{COCN}$ & 2 & $2,690 \pm \mathbf{0 , 1 3 0}$ & 3,009 & 2,837 & 2,950 & 2,950 & 2,900 & 2,354 \\
\hline $\mathbf{C}_{2} \mathbf{H}$ & 2 & $2,969 \pm \mathbf{0 , 0 6 0}$ & 3,081 & 3,015 & 3,160 & 3,173 & 3,076 & 3,128 \\
\hline $\mathbf{H S}_{2}$ & 2 & $1,907 \pm \mathbf{0 , 0 2 3}$ & 1,853 & 1,957 & 1,914 & 1,909 & 1,936 & 1,961 \\
\hline $\mathrm{HOO}$ & 2 & $1,078 \pm 0,006$ & 1,349 & 1,065 & 1,109 & 1,086 & 1,050 & 1,049 \\
\hline $\mathbf{B}_{2} \mathbf{N}$ & 2 & $3,098 \pm \mathbf{0 , 0 0 5}$ & 3,266 & 3,134 & 3,279 & 3,251 & 3,258 & 3,220 \\
\hline $\mathbf{B H}_{2}$ & 2 & 0,388 & 0,256 & 0,248 & 0,340 & 0,323 & 0,297 & 0,300 \\
\hline $\mathrm{C}$ & 1 & $1,238 \pm \mathbf{0 , 0 0 8}$ & 1,308 & 1,074 & 0,969 & 0,855 & 0,976 & 0,859 \\
\hline $\mathrm{CH}$ & 2 & $0,210 \pm \mathbf{0 , 0 1 5}$ & 0,026 & 0,083 & 0,223 & 0,179 & 0,205 & 0,182 \\
\hline $\mathbf{C H}_{2}$ & 2 & 1,127 & 1,134 & 0,962 & 0,949 & 0,929 & 0,995 & 1,003 \\
\hline $\mathrm{NH}$ & 1 & $0,370 \pm \mathbf{0 , 0 0 4}$ & 0,284 & 0,271 & 0,283 & 0,255 & 0,195 & 0,184 \\
\hline $\mathbf{N H}_{2}$ & 2 & $0,771 \pm \mathbf{0 , 0 0 5}$ & 0,823 & 0,708 & 0,772 & 0,795 & 0,697 & 0,745 \\
\hline $\mathbf{O} 2$ & 3 & $0,448 \pm \mathbf{0 , 0 0 6}$ & 0,516 & 0,449 & 0,466 & 0,420 & 0,401 & 0,370 \\
\hline $\mathrm{OH}$ & 2 & 1,828 & 1,916 & 1.766 & 1,874 & 1,879 & 1,778 & 1,790 \\
\hline HNO & 1 & $0,338 \pm 0,015$ & 0,437 & 0,320 & 0,243 & 0,193 & 0,294 & 0,271 \\
\hline $\mathrm{NO}_{2}$ & 2 & $2,273 \pm \mathbf{0 , 0 0 5}$ & 2,261 & 2,274 & 2,344 & 2,310 & 2,280 & 2,277 \\
\hline $\mathbf{S} 2$ & 3 & $1,670 \pm \mathbf{0 , 0 1 5}$ & 1,559 & 1,691 & 1,654 & 1,640 & 1,670 & 1,681 \\
\hline $\mathrm{SH}$ & 2 & $2,317 \pm \mathbf{0 , 0 0 2}$ & 2,288 & 2,371 & 2,302 & 2,310 & 2,321 & 2,348 \\
\hline $\mathrm{SO}_{2}$ & 1 & $1,107 \pm \mathbf{0 , 0 0 8}$ & 1,418 & 1,157 & 1,152 & 1,058 & 1,180 & 1,142 \\
\hline $\mathrm{PH}$ & 3 & $1,028 \pm \mathbf{0 , 0 1 0}$ & 0,895 & 0,999 & 0,957 & 0,911 & 0,981 & 0,944 \\
\hline $\mathbf{P H}_{2}$ & 2 & $1,271 \pm \mathbf{0 , 0 1 0}$ & 1,167 & 1,265 & 1,246 & 1,225 & 1,270 & 1,270 \\
\hline $\mathrm{V}_{\mathrm{m}}$ & & & $-0,065$ & 0,028 & $-0,016$ & 0,005 & 0,017 & 0,036 \\
\hline$\pm \delta_{\mathrm{N}-1}$ & & & $\pm 0,155$ & $\pm 0,085$ & $\pm 0,118$ & $\pm \mathbf{0 , 1 3 8}$ & $\pm 0,095$ & $\pm 0,128$ \\
\hline RMS & & & 0,164 & 0,090 & 0,117 & 0,135 & 0,094 & 0,131 \\
\hline
\end{tabular}

${ }^{a}$ Ref. 98.

dos elétrons onde é quase nula a probabilidade de se encontrar outro elétron, conhecido como "cusp", é de difícil representação por funções de um-elétron. Os métodos CBS resolvem este problema aplicando funções de dois-elétrons, em que a distância entre eles é tratada explicitamente, associando a estratégias como a extrapolação do conjunto de funções de base. ${ }^{46}$ Esta extrapolação também resolve outro problema, o truncamento do conjunto de funções de base, com o uso de um modelo matemático adequado para a convergência e uma sequência de conjunto de funções de base bem definidos..$^{32,47,100}$

Uma última análise pode ser feita com respeito ao método CBS-4M que gerou resultados ruins para ambas as propriedades. Neste método a otimização da geometria e o cálculo da energia do ponto zero são realizados no nível HF/6-31G*, porém, neste caso esta geometria é utilizada para os cálculos single-point de níveis superiores de teoria. Tem-se aqui dois problemas, o uso de frequências harmônicas e a geração de orbitais no nível HF. Neste nível de cálculo é realizado um tratamento matemático rigoroso para os orbitais ocupados, os orbitais virtuais gerados são muito difusos e não apropriados para um posterior tratamento de correlação eletrônica ${ }^{46}$ Outra questão é que não são realizados cálculos de nível de teoria superior ao MP4, sendo prejudicial na obtenção da energia, já que cálculos QCI ou CCSD corrigem resíduos de contaminação spin melhorando o resultado. ${ }^{6,48}$

Pela análise dos resultados para os valores estudados foi possível averiguar o método mais adequado para o estudo destas propriedades eletrônicas. É importante salientar que, com exceção do método CBS-4M, as metodologias apresentaram valores dentro da precisão aceita de $\pm 8,4 \mathrm{~kJ} / \mathrm{mol}$ para a $\mathbf{A P}$ e de $\pm 0,1 \mathrm{eV}$ para a $\mathbf{A E}$ entre o valor calculado e experimental. ${ }^{92}$

Finalmente, fez-se o estudo para o tempo médio de CPU gasto para a realização dos cálculos (Tabela 3 ). $\mathrm{O}$ cálculo para determinar o tempo de CPU foi feito para 22 sistemas $\left(\mathrm{C}_{2} \mathrm{H}_{2}, \mathrm{CH}_{3} \mathrm{COCN}, \mathrm{CH}\right.$, $\mathrm{NH}_{2}, \mathrm{OH}, \mathrm{HNO}, \mathrm{PH}_{2}, \mathrm{BH}_{3}, \mathrm{CH}_{2}, \mathrm{CH}_{3}, \mathrm{NH}, \mathrm{S}_{2}, \mathrm{SO}_{2}, \mathrm{CH}_{3} \mathrm{COOH}$, $\mathrm{NH}_{3}, \mathrm{O}_{2}, \mathrm{NO}_{2}, \mathrm{SH}, \mathrm{HOOH}, \mathrm{B}_{2} \mathrm{~N}^{-}, \mathrm{PH}$ e $\mathrm{ClCH}_{2} \mathrm{COOH}$ ), utilizando computadores com processador Intel Pentium 4, com clock de 1,60 GHz e memória RAM de $1023 \mathrm{MB}$. 
Tabela 3. Valores do tempo de CPU médio (em segundos) para 22 sistemas estudados com os métodos CBS-4M, CBS-QB3, G2, G2(MP2), G3 e G3(MP2)

\begin{tabular}{lcccccc}
\hline & CBS-4M & CBS-QB3 & G2 & G2(MP2) & G3 & G3(MP2) \\
\hline $\mathrm{T}_{\mathrm{CPU}}$ médio & 201,59 & 919,77 & 2145,49 & 725,13 & 1507,12 & 465,36 \\
\hline
\end{tabular}

Observa-se a seguinte ordem crescente para o valor do tempo de CPU médio: CBS-4M < G3(MP2) < G2(MP2) < CBS-QB3 < G3 < G2. Dessa forma, apesar de os métodos CBS-4M, G3(MP2) e G2(MP2) terem apresentado os menores valores de tempo gasto, eles perdem em informação gerando os maiores valores de desvio padrão e de RMS em relação às outras metodologias.

\section{$O$ custo computacional dos métodos compostos}

Em relação ao custo computacional o método CBS-4M foi o mais eficiente, as principais características que possibilitam este resultado são o uso de conjuntos de funções de base menores para altos níveis de teoria, por otimizar a geometria no nível HF/6-31G* e por não realizar cálculos com nível de teoria superior ao MP4, ou seja, níveis de teoria QCI ou CCSD. Para os métodos G3(MP2) ${ }^{4}$ e G2(MP2) ${ }^{2}$ o nível de teoria MP4 é substituído pelo MP2. Para se obter a energia são necessários somente dois cálculos single-point: QCISD(T)/6-31G(d) e MP2(FC)/6-311++G(2df,2p) para G3(MP2) e QCISD(T)/6-31G(d) e MP2/6-311+G(3df,2p) para G2(MP2), isto diminui o tempo gasto de CPU.

O método CBS-QB3 foi o quarto melhor método para os tempos de CPU, sendo 39\% mais eficiente que o método G3 e 57\% mais eficiente que o método G2. Os dois últimos geraram os respectivos custos computacionais por reotimizarem a geometria, obtida primeiramente no nível HF/6-31G(d), no nível MP2/6-31G(d) considerando os elétrons da camada interna. Outro fator é a consideração do truncamento da base utilizando o cálculo no nível MP2 com todos os elétrons e com um conjunto de funções de base grande - 6-311++G(2df,2p). ${ }^{3}$ Os conjuntos de funções de base no método G3 para níveis superiores de teoria são menores em relação ao G2, por isso a ordem observada para ambos.

Uma última consideração é com relação ao "cusp" intereletrônico. Como já observado, os métodos CBS consideram-no aplicando funções de dois-elétrons e um processo adequado de extrapolação do conjunto de funções de base. Na equação da energia obtida com a extrapolação há um termo, chamado de fator de interferência (o mesmo da Equação 9), que tem o efeito de acelerar a convergência das energias pares de segunda ordem nos cálculos CCSD, isto favorece sobremaneira uma convergência rápida e com valores calculados confiáveis (menos o CBS-4M que devido a outros fatores já discutidos não segue esta regra). Portanto, caso a reação estudada apresente moléculas semelhantes às que foram analisadas é preferível utilizar a metodologia CBS-QB3 devido ao melhor custo computacional e por gerar bons resultados no estudo das propriedades termodinâmicas.

\section{CONCLUSÃO}

O resultado da validação através da análise estatística do valor médio, do desvio padrão e do valor quadrático médio, bem como dos valores médios para o tempo de CPU gasto para realizar os cálculos, mostrou que o método CBS-QB3 foi a metodologia mais adequada dentre as testadas para os sistemas estudados neste trabalho.

A confiabilidade e eficiência do método CBS-QB3 demonstra sua utilidade no estudo de diversos parâmetros físico-químicos, os quais permitem realizar uma ampla análise de como se processam as alterações estruturais e energéticas resultantes de uma reação química. A abordagem teórica permite fornecer respostas sobre os pontos não explorados experimentalmente, ou mesmo onde a parte experimental oferece resultados insuficientes para uma abordagem mais completa do problema.

Como estudamos neste artigo propriedades que envolvem diferenças de energia, um trabalho envolvendo sistemas análogos presentes em reações químicas está em andamento, inclusive com a descrição do cálculo da energia molecular de estados de transição através do método composto CBS-QB3.

\section{AGRADECIMENTOS}

Às agências CNPq e FAPESP pelo apoio financeiro.

\section{REFERÊNCIAS E NOTAS}

1. Curtiss, L. A.; Raghavachari, K.; Truks, G. W.; Pople, A. J.; J. Chem. Phys. 1991, 94, 7221.

2. Curtiss, L. A.; Raghavachari, K.; Pople, A. J.; J. Chem. Phys. 1993, 98 , 1293.

3. Curtiss, L. A.; Raghavachari, K.; Redfern, P. C.; Rassolov, V.; Pople, A. J.; J. Chem. Phys. 1998, 109, 7764.

4. Curtiss, L. A.; Redfern, P. C.; Raghavachari, K.; Rassolov, V.; Pople, A. J.; J. Chem. Phys. 1999, 110, 4703.

5. Montgomery Jr., J. A.; Frisch, M. J.; Ochterski, J. W.; Petersson, G. A.; J. Chem. Phys. 1999, 110, 2822.

6. Montgomery Jr., J. A.; Frisch, M. J.; Ochterski, J. W.; Petersson, G. A.; J. Chem. Phys. 2000, 112, 6532.

7. Sun, W.; Saeys, M.; J. Phys. Chem. A 2008, 112, 6918.

8. Aguilera-Iparraguirre, J.; Curran, H. J.; Klopper, W.; Simmie, J. M.; J. Phys. Chem. A 2008, 112, 7047.

9. Lv, C. L.; Liu, Y. D.; Zhong, R.; J. Phys. Chem. A 2008, 112, 7098.

10. Ess, D. H.; Houk, K. N.; J. Phys. Chem. A 2005, 109, 9542.

11. Coote, M. L.; Krenske, E. H.; Maulana, I.; Steinbach, J.; Wild, S. B.; Heteroatom Chemistry 2008, 19, 178.

12. Yang, L.; Liu, J.; Wang, L.; He, H.; Wang, Y.; Li, Z.; J. Comput. Chem. 2008, 29, 550.

13. Landera, A.; Mebel, A. M.; Kaiser, R. I.; Chem. Phys. Lett. 2008, 459, 54.

14. Qi, Y.; Feng, D.; Feng, S.; J. Molec. Struc. Theochem 2008, 856, 96.

15. Mousavipour, S. H.; Saheb, V.; Bull. Chem. Soc. Jpn. 2007, 80, 1901.

16. Vandeputte, A. G.; Sabbe, M. K.; Reyniers, M.; Speybroeck, V. V.; Waroquier, M.; Marin, G. B.; J. Phys. Chem. A 2007, 111, 11771.

17. Range, K.; Riccardi, D.; Cui, Q.; Elstner, M.; York, D. M.; Phys. Chem. Chem. Phys. 2005, 7, 3070.

18. Azenkeng, A.; Laumb, J. D.; Jensen, R. R.; Olson, E. S.; Benson, S. A.; Hoffmann, M. R.; J. Phys. Chem. A 2008, 112, 5269.

19. Frash, M. V.; Hopkinson, A. C.; Bohme, D. K.; J. Phys. Chem. A 1999, 103,7872 .

20. Range, K.; López, C. S.; Moser, A.; York, D. M.; J. Phys. Chem. A 2006, 110, 791.

21. Kabli, S.; Beelen, E. S. E.; Ingemann, S.; Heriksen, L.; Hammerum, S.; Int. J. Mass Spectrom. 2006, 249-250, 370.

22. Richard, R. M.; Ball, D. W.; J. Molec. Model. 2008, 14, 21.

23. Taskinen, A.; Nieminen, V.; Toukoniitty, E.; Muzin, D. Y.; Hotokka, M.; Tetrahedron 2005, 61, 8109.

24. Wang, L.; Int. J. Mass Spectrom. 2007, 264, 84.

25. Jalbout, A. F.; Jalbout, F. N.; Alkahby, H. Y.; J. Molec. Struc. Theochem 2001, 574, 141 .

26. Miller, T. M.; Viggiano, A. A.; Dolbier, W. R. J.; Sergeeva, T. A.; Friedman, J. F.; J. Phys. Chem. A 2007, 111, 1024.

27. Namazian, M.; Siahrostami, S.; Coote, M. L.; J. Fluorine Chem. 2008, 129, 222 
28. Toth, A. M.; Liptak, M. D.; Phillips, D. L.; Shields, G. C.; J. Chem. Phys. 2001, 114, 4595.

29. Sun, H.; Tan, N.; He, H.; Pan, X.; Su, Z.; Wang, R.; Theor. Chem. Account 2008, 119, 501.

30. Doren, J. M. V.; Miller, T. M.; Viggiano, A. A.; J. Chem. Phys. 2008, 128,094310

31. Doren, J. M. V.; Miller, T. M.; Viggiano, A. A.; J. Phys. Chem. A 2007, 111, 13595.

32. Klopper, K.; Bak, K. L.; Jørgensen, P.; Olsen, J.; Helgaker, T.; J. Phys. B 1999, 32, R103.

33. Fry, A. J.; Tetrahedron 2008, 64, 2101.

34. Nunes, P. M.; Estácio, S. G.; Lopes, G. T.; Cabral, B. J. C.; Santos, R. M. B.; Simões, J. A. M.; Org. Lett. 2008, 10, 1613.

35. Simmie, J. M.; Black, G.; Curran, H. J.; Hinde, J. P.; J. Phys. Chem. A 2008, 112,5010

36. Wei, Y.; Singer, T.; Mayr, H.; Sastry, G. N.; Zipse, H.; J. Comput. Chem. 2008, 29, 291.

37. Tu, C.; Guo, W.; Hu, C.; J. Phys. Chem. A 2008, 112, 117.

38. Duchowicz, P. R.; Cobos, C. J.; J. Phys. Chem. A 2008, 112, 6198.

39. Badenes, M. P.; Cobos, C. J.; J. Molec. Struc. Theochem 2008, 856, 59.

40. Denis, P. A.; J. Phys. Chem. A 2004, 108, 11092.

41. Richard, R. M.; Ball, D. W.; J. Molec. Struc. Theochem 2006, 776, 89.

42. Bond, D.; J. Org. Chem. 2007, 72, 5555.

43. Couto, P. C.; Cabral, B. J. C.; Simões, J. A. M.; Chem. Phys. Lett. 2006, 419, 486.

44. Pickard IV, F. C.; Pokon, E. K.; Liptak, M. D.; Shields, G. C.; J. Chem. Phys. 2005, 122, 024302.

45. Vessechi, R.; Galembeck, S. E.; Lopes, N. P.; Nascimento, P. G. B. D.; Crotti, A. E. M.; Quim. Nova 2008, 31, 840.

46. Laschuk, E. F.; Livotto, P. R. Em Métodos de Química Teórica e Modelagem Molecular; Morgon, N. H.; Coutinho, K., eds.; Editora Livraria da Física: São Paulo, 2007, cap. 9.

47. Petersson, G. A. Em Quantum-Mechanical Prediction of Thermochemical Data; Cioslowski, J., ed.; Kluwer Academic Publisgers: Dordrecht, 2001, cap. 4.

48. Ochterski, J. W.; Petersson, G. A.; Montgomery Jr., J. A.; J. Chem. Phys. 1996, 104, 2598

49. Helgaker, T.; Jørgensen, P.; Olsen, J.; Molecular Electronic-Structure Theory; John Wiley \& Sons, LTD: New York, 2000.

50. Curtiss, L. A.; Carpenter, J. E.; Raghavachari, K.; Pople, A. J.; J. Chem. Phys. 1992, 96, 9030.

51. Para o conjunto de funções de base no método CBS-QB3 é utilizada uma notação específica por Montgomery et al. (ref. 5). Por exemplo, 6-311G $(2 d, d, p)$ significa que para o segundo período são aplicadas duas funções $d$, para o primeiro período uma função $d$ e para os hidrogênios uma função de polarização $p \cdot 6-31+G((d) f, p)$ significa que é aplicada uma função $d$ para ambos primeiro e segundo períodos, incluindo uma função $f$ para este último, e uma função $p$ para os hidrogênios.

52. Schwartz, C.; Phys. Rev. 1962, 126, 1015

53. Petersson, G. A.; Nyden, M. R.; J. Chem. Phys. 1981, 75, 3423.

54. Nyden, M. R.; Petersson, G. A.; J. Chem. Phys. 1981, 75, 1843.

55. Petersson, G. A.; Licht, S. L.; J. Chem. Phys. 1981, 75, 4556.

56. A unidade em mili-hartree do coeficiente empírico aparece na equação da ref. 5 como mEh.

57. Pople, J. A.; Beveridge, D. L.; Approximate Molecular Orbital Theory; McGraw-Hill Book Company: New York, 1970.

58. Pople, J. A.; Schleyer, P. V. R.; Radom, L.; Hehre, W. J.; Ab Initio Molecular Orbital Theory, Wiley-Interscience Publication John Wiley \& Sons: USA, 1986.

59. Petersson, G. A.; Al-Laham, M. A.; J. Chem. Phys. 1991, 94, 6081.

60. Rienstra-Kiracofe, J. C.; Tschumper, G. S.; Schaefer, H. F.; Nandi, S.; Ellison, G. B.; Chem. Rev. 2002, 102, 231.

61. Hotop, H.; Lineberger, W. C.; J. Phys. Chem. Ref. Data 1975, 4, 539.
62. Hotop, H.; Lineberger, W. C.; J. Phys. Chem. Ref. Data 1985, 14, 731. 63. Ervin, K. M.; Chem. Rev. 2001, 101, 391.

64. Reimers, J. R.; Ulstrup, J.; Meyer, T. J.; Solomon, G. C.; Chem. Phys. 2006, 324, 1.

65. Lowe, J. P.; J. Am. Chem. Soc. 1977, 99, 5557.

66. Knighton, W. B.; Grimsrud, E. P.; J. Am. Chem. Soc. 1992, 114, 2336.

67. Bensasson, R. V.; Zoete, V.; Dinkova-Kostova, A. T.; Talalay, P.; Chem. Res. Toxicol. 2008, 21, 805.

68. Librando, V.; Alparone, A.; Tomaselli, G.; J. Molec. Model. 2008, 12, 489.

69. Alexandratos, S. D.; Zhu, X.; Inorg. Chem. 2008, 47, 2831.

70. Viggiano, A. A.; Miller, T. M.; J. Chem. Phys. 2007, 127, 244305.

71. Bai, Q.; Song, B.; Hou, J.; He, P.; Phys. Lett. 2008, 372, 4545.

72. Mawhinney, R. C.; Goddard, J. D.; J. Molec. Struc. Theochem 2008, $856,16$.

73. Jin, H.; Dai, Y.; Long, R.; Guo, M.; Huang, B.; Zhang, R.; J. Chem. Phys. 2008, 128, 114710.

74. Bhattacharya, S. K.; Kshirsagar, A.; Eur. Phys. J. D 2008, 48, 355.

75. Reinard, M. S.; Johnston, M. V.; J. Am. Soc. Mass Spectrom. 2008, 19, 389.

76. Taylor, T. R.; Asmis, K. R.; Xu, C.; Neumark, D. M.; Chem. Phys. Lett. 1998, 297, 133

77. http://www.chem.qmul.ac.uk/iupac, IUPAC - Glossary of terms in Physical Organic Chemistry, acessada em Novembro 2009.

78. Lias, S. G.; Liebman, J. F.; Levin, R. D.; J. Phys. Chem. Ref. Data 1984, 13, 695.

79. Hunter, E. P. L.; Lias, S. G.; J. Phys. Chem. Ref. Data 1998, 27, 413.

80. DeFrees, D. J.; McLean, A. D.; J. Comput. Chem. 2001, 101, 391.

81. http://www.hbcpnetbase.com, Handbook of Chemistry and Physics, CRC, 88th, 2007-2008, acessada em Novembro 2009.

82. Higashi, N.; Tanimoto, K.; Nishioka, M.; Ishikawa, K.; Taya, M.; J. Biochem. 2008, 144, 77.

83. Morgan, J. E.; Gennis, R. B.; Maeda, A.; Photochem. Photobiol. 2008, 84, 1038.

84. Zhou, L.; Singleton, C.; Le Brun, N. E.; Biochem. J. 2008, 413, 459.

85. He, N.; Xie, H.; Ding, Y.; Microporous Mesoporous Mater. 2008, 111, 551.

86. Nielsen, U. G.; Majzlan, J.; Grey, C. P.; Chem. Mater. 2008, 20, 2234.

87. Hiemstra, T.; Riemsdijk, W. H.; Geochim. Cosmochim. Acta 2007, 71, 5913.

88. Yang, G.; Zu, Y.; Liu, C.; Fu, Y.; Zhou, L.; J. Phys. Chem. B 2008, 112, 7104.

89. Robb, D. B.; Smith, D. R.; Blades, M. W.; J. Am. Soc. Mass Spectrom. 2008, 19, 955.

90. Macht, J.; Janik, M. J.; Neurock, M.; Iglesia, E.; J. Am. Chem. Soc. 2008, 130, 10369.

91. http://mathworld.wolfram.com/topics/ErrorAnalysis.html, acessada em Novembro 2009; Weisstein, E. W. MathWorld - A Wolfram Web Resource - Error Analysis, 2007.

92. Pople, A. J.; Head-Gordon, M.; Fox, D. J.; Raghavachari, K.; Curtiss, L. A.; J. Chem. Phys. 1989, 90, 5622.

93. Frisch, M. J.; Trucks, G. W.; Schlegel, H. B.; Scuseria, G. E.; Robb, M. A.; Cheeseman, J. R.; Zakrzewski, V. G.; Montgomery, J. A. J.; Stratmann, R. E.; Burant, J. C.; Dapprich, S.; Millam, J. M.; Daniels, A. D.; Kudin, K. N.; Strain, M. C.; Farkas, O.; Tomasi, J.; Barone, V.; Cossi, M.; Cammi, R.; Mennucci, B.; Pomelli, C.; Adamo, C.; Clifford, S.; Ochterski, J.; Petersson, G. A.; Ayala, P. Y.; Cui, Q.; Morokuma, K.; Malick, D. K.; Rabuck, A. D.; Raghavachari, K.; Foresman, J. B.; Cioslowski, J.; Ortiz, J. V.; Baboul, A. G.; Stefanov, B. B.; Liu, G.; Liashenko, A.; Piskorz, P.; Komaromi, I.; Gomperts, R.; Martin, R. L.; Fox, D. J.; Keith, T.; Al-Laham, M. A.; Peng, C. Y.; Nanayakkara, A.; Gonzalez, C.; Challacombe, M.; Gill, P. M. W.; Johnson, B. G.; Chen, W.; Wong, M. W.; Andres, J. L.; Head-Gordon, M.; Replogle, E. S.; Pople, J. A.; Gaussian 98, Gaussian, Inc., Wallingford, CT, 1998.

94. Frisch, M. J.; Trucks, G. W.; Schlegel, H. B.; Scuseria, G. E.; Robb, M. A.; Cheeseman, J. R.; Montgomery, A., J.; Vreven, T.; Kudin, K. N.; 
Burant, J. C.; Millam, J. M.; Iyengar, S. S.; Tomasi, J.; Barone, V.; Mennucci, B.; Cossi, M.; Scalmani, G.; Rega, N.; Petersson, G. A.; Nakatsuji, H.; Hada, M.; Ehara, M.; Toyota, K.; Fukuda, R.; Hasegawa, J.; Ishida, M.; Nakajima, T.; Honda, Y.; Kitao, O.; Nakai, H.; Klene, M.; Li, X.; Knox, J. E.; Hratchian, H. P.; Cross, J. B.; Bakken, V.; Adamo, C.; Jaramillo, J.; Gomperts, R.; Stratmann, R. E.; Yazyev, O.; Austin, A. J.; Cammi, R.; Pomelli, C.; Ochterski, J. W.; Ayala, P. Y.; Morokuma, K.; Voth, G. A.; Salvador, P.; Dannenberg, J. J.; Zakrzewski, V. G.; Dapprich, S.; Daniels, A. D.; Strain, M. C.; Farkas, O.; Malick, D. K.; Rabuck, A. D.; Raghavachari, K.; Foresman, J. B.; Ortiz, J. V.; Cui, Q.; Baboul, A. G.; Clifford, S.; Cioslowski, J.; Stefanov, B. B.; Liu, G.; Liashenko, A.; Piskorz, P.; Komaromi, I.; Martin, R. L.; Fox, D. J.; Keith, T.; Al-Laham, M. A.; Peng, C. Y.; Nanayakkara, A.; Challacombe, M.; Gill, P. M. W.; Johnson, B.; Chen, W.; Wong, M. W.; Gonzalez, C.; Pople, J. A.; Gaussian 03, Gaussian, Inc., Wallingford, CT, 2004.
95. Travers, M. J.; Cowles, D. C.; Ellison, G. B.; Chem. Phys. Lett. 1989, $164,449$.

96. Asmis, K. R.; Taylor, T. R.; Neumark, D. M. J. Chem. Phys. 1999, 111, 10491.

97. Nimlos, M. R.; Ellison, G. B.; J. Phys. Chem. 1986, 90, 2574.

98. Eds. Linstrom, P. J.; Mallard, W. G. NIST Chemistry WebBook - National Institute of Standards and Technology, Number 69; Gaithersburg MD, 20899: http://webbook.nist.gov, acessada em Novembro 2009.

99. Montgomery, J. A.; Ochterski, J. W.; Petersson, G. A.; J. Chem. Phys. 1994, 101, 5900.

100. Lima, J. C. B.; Dissertação de Mestrado, Universidade Estadual de Campinas, Brasil, 2008. 